\title{
Comprehensive Study on Software Defined Network for Energy Conservation
}

\author{
Dr. Mohammed Najm Abdulla ${ }^{1}$, Bassam Noori Shaker ${ }^{2}$ \\ Computer Engineering Dept, University of Technology, $\operatorname{Iraq}^{1}$ \\ Institute for Postgraduate Studies, UITC, $\operatorname{Iraq}^{2}$
}

\begin{abstract}
SDN is another NW topology that gives more encourage to the overseer for dealing with the entire NW (administrations and resources) by decoupling the control plane from the information arrange and concentrate the control coherently .in this papers we presented. A comprehensive view of the SDN begins with definition then primary standards of SDN. Next, we illuminated the fundamental segments of the SDN architecture and how these segments communicate with each either. Next, we introduced ideas about the energy conservation in SDN environment and we discussed the virtualization and its significance from the perspective of the SDN.
\end{abstract}

Keywords: SDN, virtualization, openflow, NFV, TCAM, energy saving.

\section{INTRODUCTION}

Computer networks are traditionally constructed from And this can be done by centralized network control, enormous number of networking devices such as (routers, which is can be obtain as the controller of this switches) and many sophisticated protocols which are architectural (control plane) which responsible of embedded in them[1] [2] [3] [4]. Packet forwarding Coordinate intermediate and facilitates the communication decisions are made by network devices individually, based between higher level of application (application plane) and on suitable high-level network policies ,the process of the lower level of network element (infrastructure layer) tuning the configuration for network devices should be and as show in the figure (1).

make separately by the network operators by using lowlevel commands so reconfiguration the network for any reason will be very difficult and error - prone tasks[5].

Changing network functionality to meet user requirements needs to enforce new policies within network devices so the traditional networks don't offer a dynamic way to provide user requirements.

Software defined network (SDN)[6] [7] is a new network architecture that enable us from building an efficient network without disadvantage of traditional architecture , by stripping the control from the forwarding devices and make the control logically centralized, this founded new planes one for the control decision which is called control plane and the other data plane that contain simple forwarding devices, the network based on the SDN architecture can be programmable by software applications running over the control plane[8].

This papers review most important aspects of this new architecture, and clarify advantage over the traditional network paradigm and how it will be useful in energy saving.

\section{SDN DEFINITION}

According to the [9] Software defined network (SDN) is architectural approach that simplifies and control Network operations through close interaction between the applications and network services and devices, both $\mathrm{Be}$ real or virtual.

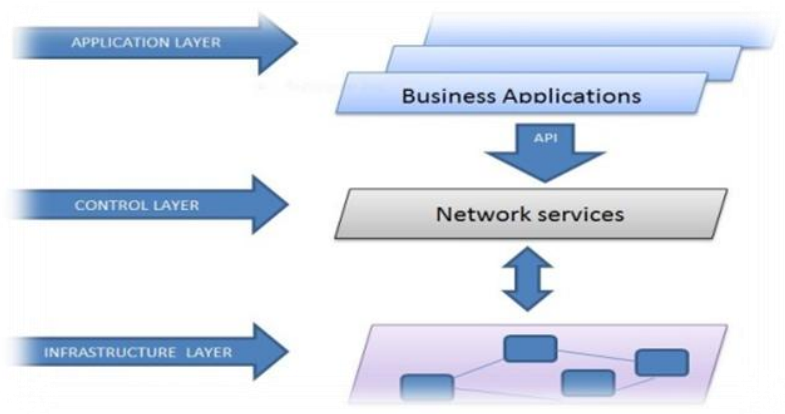

Fig.1. general view of SDN layers

\section{SDN ARCHITECTURE COMPONENTS}

According to the general definition of the architecture, next we will clarify the main components of the SDN and the function of each of them and how these components are communicate with each other as show in figure (2).

\section{A. SDN Inferastructure}

SDN infrastructure (physically networking) such as in the traditional network, is consist of network devices (e.g. switches, routers) but they become much simpler because of stripping the control from them[10] [6]. These elements are connected with the controller via Southbound interface (southbound APIs).The main function of Southbound APIs are support control over the network and facilitate the SDN Controller to dynamically make changes . 


\section{B. SDN controller(Controll Plane)}

is the middle point between the higher component ( NW application ) and the lower one ( physical network ) and is represent a logically centralized network which responsible of translating the needs of the applications network down to the SDN infrastructure and provide the network applications with an abstract view of the NW.

SDN controller communicates with applications and services over the NW via Northbound interface APIs. Northbound interface APIs unable the NW operators to modify or customize their NW and without it all network applications must come directly from devices vendors.

Network hypervisors is a layer of software that placed between the hardware and the one or more virtual machines that it supports in case of SDN hypervisor enable different virtual machine to share the same HW resources .

\section{Network Applications(Application Plane)}

set of programs that explicitly, directly, and programmatically communicate their network requirements and desired network behavior to the SDN Controller via Northbound interface such as business applications[11] [12].
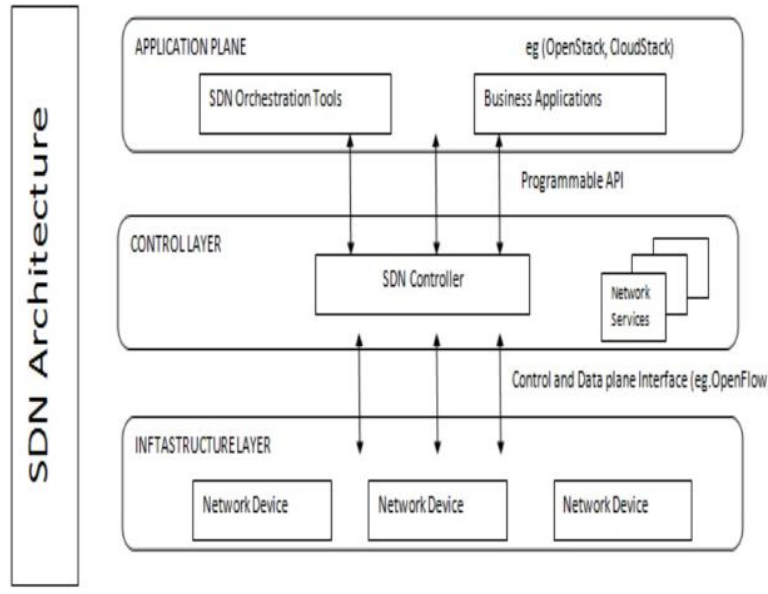

Fig.2. SDN architecture components

\section{OPEN FLOW}

Open flow is the earliest SDN protocol that defined between the control and data plane [13] [14].Openflow enable the network application in management plane to access to data plane of the network devices ( switches or routers ) so the control programs don't need to cooperate with the network infrastructure devices. Openflow is predefined match rules -based and this rules can be programmed either statically or dynamically by the SDN control software.

The process of communication between the switch and control is message - based [15]. Where set of messages that already been defined will exchange between both side of openflow protocol via a secure channel.
There are three type of openflow messages :

A. Control to switch messages : started from the control towards the switch and used for check and administrate the state of the switch and these messages not necessarily get a feedback from the switch.

B. Asynchronous messages : started from the switch and used for notify the controller to the event or switch changes such as enunciation the controller with packet arrival.

C. Symmetric messages : this type of messages send without request from either direction such as Echo request / replay messages, Hello messages.

\section{HOST TO HOST CONNECTION PROCESS OVER SDN/OPENFLOW NETWORK}

To demonstrate the idea of connection between two hasts over SDN/openflow network we begin with a sample scenario consist of two host and one openflow switch and one controller as show in the figure (3).

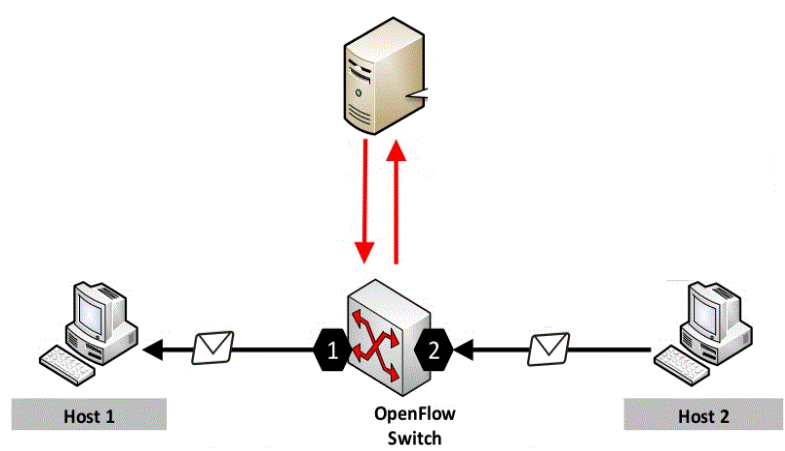

Fig.3. two hosts connection over SDN/openflow network.

When the host 1 ( client) want to initiate a connection with the host 2 over the SDN he will send the first packets that involved with flow for determining if the forwarding decision under control of the openflow switch or the switch need to take the decision from the control if the decision belong to the controller then a connection will setup over a secure channel via control protocol the allowing of the flow is decided by the controller based on set of prior polices if it ok then the flow details could get in to the connection table of the controller. The flow remove from the table when the openflow switch tell the controller a flow is no longer active.

There are benefits for centralizing control on the connection requests, some of undesired connection request could be dropped such as denial of service attacks, based on configurable policies in the controller[16] .

\section{KEY DIFFERENTS BETWEEN TRADITIONAL VS. SDN NETWORK ARCHITECTURE}

This section will introduce the most differences between traditional ( distributed control and middle boxes ) and modern SDN( decoupling control and data plane) network architecture based on two approaches network forwarding network devices and network applications, 
Vol. 5, Issue 9, September 2016

figure (4) show the main differences that stand on the decoupling idea.

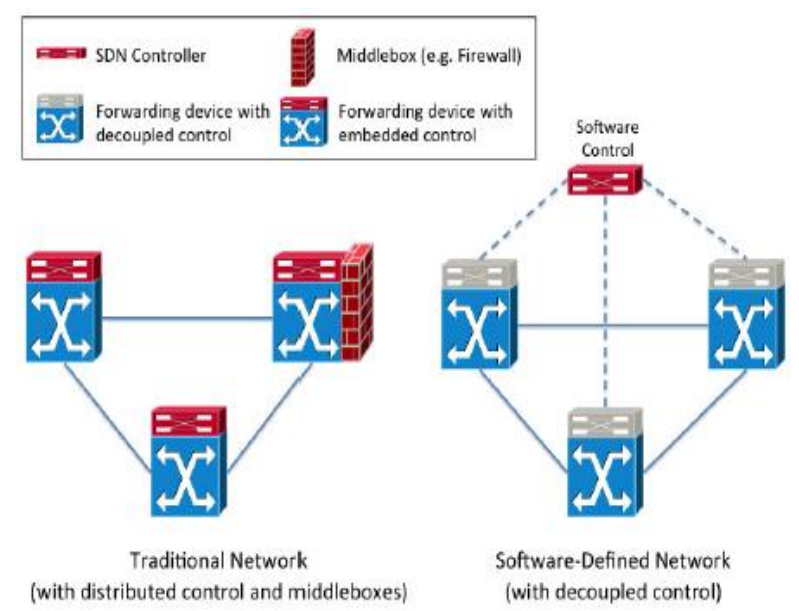

Fig.4. main differences that stand on the decoupling idea

\section{A. network forwarding devices}

with traditional approach of computer network, a computer network consist from number of NW devices ( switches, routers), network functionalities such as control decision are implemented in these network appliances, most of the functionalities are enforced in dedicated hardware in side them like associate ASIC (Application Specific Integrated Circuit). Below List of some characteristics of traditional network appliances:

- Slowly development to ASIC that provide network functionality

- Appliance (switch or router) is configured individually.

- ASIC functionality evolution is under the control of the appliance's supplier .

- Very difficult ( time consuming and error prone) doing task such as provisioning and change management .

- The appliances(switches, routers ) are proprietary.

Where as in the SDN approaches all of the control functions of a conventional switch (i.e., routing protocols ) are run in the central controller. The functionalities in the switches are limited entirely to the data plane.

\section{B. Network applications}

- Conventional network applications only implicitly describe their NW requirements, typically include several human processing steps, e.g., to negotiate if there is sufficient resource and policy control to support the application. Whereas with the SDN explicitly links network control to each application's functional requirements.

- conventional networks do not show information and network state to the applications using them. SDN NW applications can monitor network events and adapt accordingly.

- Traditional networks do not offer a (dynamic) way to express most of user requirements such as throughput (15)(the rate of successful message delivery over a communication channel) while with SDN these requirement can be achieve dynamically.

\section{ROLE OF VIRTUALIZATION IN SDN}

The general concept of virtualization is stripping logical function from underlying physical infrastructure[17] [18] . There are many face of virtualization such as storage virtualization (e.g. converting of physical disk addresses into logical block addresses for use by the OS' file system) and server virtualization which is run multiple virtual servers on the same physical server so each virtual server machine (VM) has illusion it running as physical server (e.g. virtual Web servers is used Instead of requiring a disjoint computers for each Web server, many of virtual servers can build on the same computer) and network virtualization (NV) which is running multiple virtual network on the same network infrastructure so each one of them has illusion it running as a physical network ( e.g. VLAN)[19]. And as show in the figure (5).

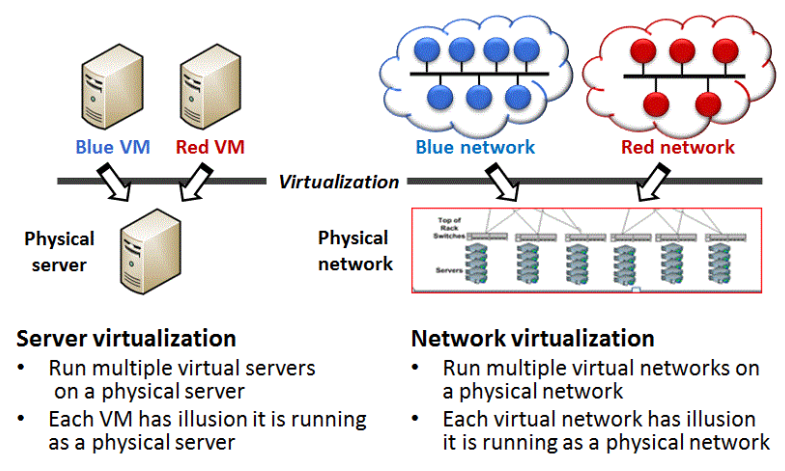

Fig.5. two types of virtualization server \& network.

So according to the definition of the SDN that mention early in (II) we can understanding The key idea behind the SDN architecture is the concept of integration between network and the virtualization and this will enabling to reduce both a cost of a hardware and limitation in network configuration.

\section{A. Solving NW problem based on NFV and SDN}

The traditional network infrastructures are suffering from many problems as mention early in (VI)such as higher cost and propriety hardware and energy consumptions, so any new solution for this problems should be focuses on two key

- Lower cost and industry standard platform that support communication functionality.

- Transform to the software driven NW architecture that support flexibility and rapidly invention .

Two software technologies are leading the solution : Network functions Virtualization (NFV) [20] [21].which increase network resource utilization in the same way reducing the cost of developing and deploying new services ,and software Defined Network(SDN) which help 
Vol. 5, Issue 9, September 2016

to construct and mange efficiently large and complex networks .in the next topic we will focused on solving energy conservation problem based on SDN architecture

VIII. POWER MANAGEMENT METHODOLOGIES OF COMPUTER NETWORK

In the computer network, approaches methodologies of power management are derived from enhancing strategies that already exist in processors of the network hardware devices which are special computer hardware [22] [23]. There are three classes of methodologies smart stand by, re-engineering and dynamic adaption :

- Re-engineering : this class of power management based on founding a new energy-saving technologies and exploit them in network architecture such as memories.

- Dynamic adaption : this class depending on modifying the services quality based on existence traffic load . and this can be achieve by two techniques Adaptive rat (AR) and Low Power Idle ( LPI) .

The idea of Adaptive rate is trade-offs between power consumption and performance (minimize device working rate dynamically by regulation the processing engine frequency). Low Power Idle, on the other hand, switching off sub component of the network when it is not used and return back to service when the system need them.

- Sleep /standby approaches doing lower standby mode for some selected unused devices in the network and if it's necessary wake up them again.

Losing network connection during the sleep mode so the appliance cannot be seen represent the main issue with this approaches because it require re-connection phase in case of wake-up again and this represent more lost time

\section{A. Energy Consumptions over SDN and NVF}

Reducing the energy in Information and Communication Technology systems [24] [25] . will faced divers challenges because this system composed of different network parts with different set of technologies. There are set of methodologies that are used as base for any reduction energy technique in the computer network, in this chapter we will examined these methodologies with SDN network architecture as case study which is a new NW architecture based on separation control from the forwarding devices and who this architecture will be fruitfully in energy - aware consuming .

\section{B. Role of SDN in Energy Saving}

One of the most important aspact future role of SDN is energy saving .many researchs focused on this aspact such as:

- A hard ware energy saving solution

TCAM (ternary Content Addressable Memory ) is an electronic circuit that is used for saving switch information such as pre-defined rule in case of openflow switch . Most power consumption in the switch came from the operations of the TCAM which are search (lookup) and insert (write).

TCAM venders design the memory as contiguous same size of blocks these blocks used for saving the information so for any incoming packet matching operation will arise and all blocks will activate for this operation. Author in [26] suppose a smart pre_classifier technique that lead for energy saving by reducing the active blocks(more active bloke more power consumption) so TCAM blocks will use pre-classifier for consulting at the first and then matching a lower number of blocks this will help in reducing the number of active block that needed and consequently reducing the power consumption as show in the fig (6).
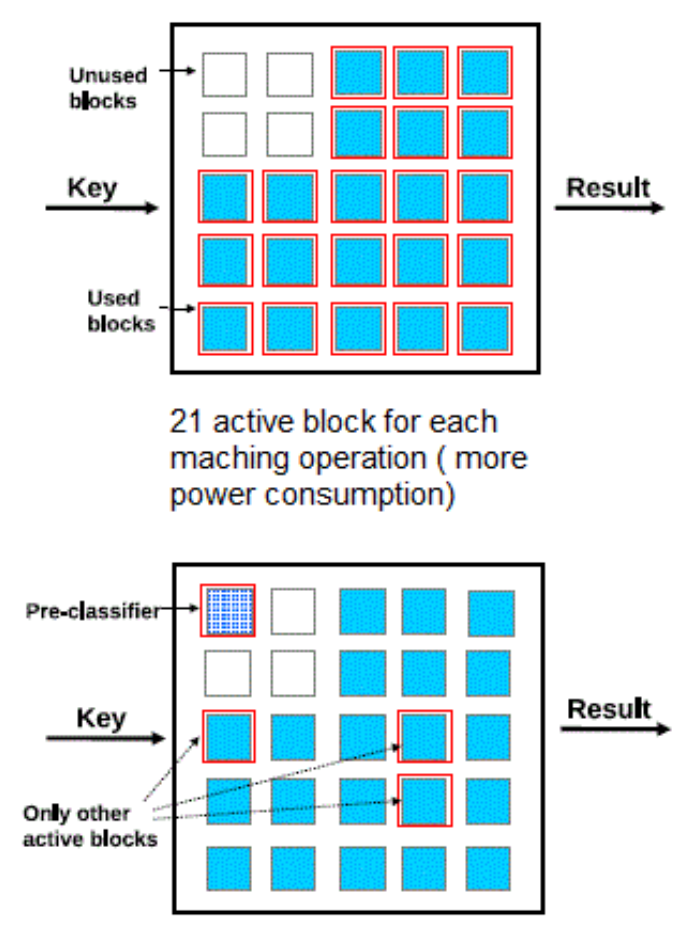

3 active block based on pre-classification tech. (less power consumption)

Fig.6.over view of per-classification tech.

Were as in[27] the author investment compacting the TCAM for minimizing the size of flow entries in open flow switches this minimizing will help in reduction of power consumption due to less search in TCAM blocks.

- Traffic-routing modification for energy saving.

This method is based on the fact that most network devices (switches and routers) are less effective in nonpeak time so according to the links capacity we can redirect the flows faraway of candidate switches and for turning off them for saving energy.

The authors in [28] [29] suppose using fat tree topology as show in figure (7) for saving energy in data centre(DC). 


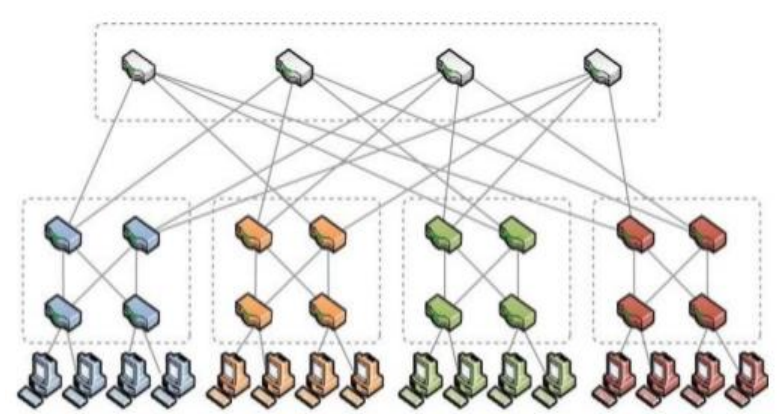

Fig.7. fat tree topology (20 switches 10 host $)$

Based on traffic-routing modification method, DC topology (fat tree) can be Expansion and contraction for saving energy about $50 \%$ of total power consumption. With the SDN we can modify DC topology easily at real time.

There are set of restriction to determine number of active switches:

- Link capacity restriction.

- Traffic demand restriction.

- TCAM size.

\section{CONCLUSION}

In this paper, we present study for the new computer network architecture (SDN) from many perspectives, its benefit and clarify SDN role in energy saving based on power management methodology. We introduce two methods for this purpose first one depending on hardware solution (adjustment TCAM saving mechanize) and the other, traffic -routing modification solution.

\section{REFERENCES}

[1] D. J. W. Anderw S. Tanenbaum "Computer Network", 5 ed., 2011.

[2] K. W. R. James F. Kurose "Computer networking a Top-Down Approach", 6 ed., 2013.

[3] S. G. Y. Navaneeth Krishnan, ""Performance Analysis of OSPF and EIGRP Routing Protocols for Greener Internetworking"," 2013.

[4] J. J. Piotr Gaj, Max Felser, "Computer Communication within Industrial Distributed Environment-a Survey"," IEEE TRANSACTIONS ON INDUSTRIAL INFORMATICS, vol. 9, no. $1,2013$.

[5] B. S. D. Larry L. Peterson Computer Networks a Systems Approach, 3 ed., 2003.

[6] C. B. Paul Göransson Software Defined Networks a Comprehensive Approach, 2014.

[7] Y. W. Wenfeng Xia, Chuan Heng Foh,Dusit Niyato,Haiyong Xie, "A Survey on Software-Defined Networking," IEEE COMMUNICATION SURVEYS \& TUTORIALS, vol. 17, no. 1, 2015.

[8] M. D. D. F. Celio Trois, Luis C. E. de Bona,Magnos Martinello, "A Survey on SDN Programming Languages:Towards a Taxonomy",’ IEEE Communications Surveys \& Tutorials, 2015.

[9] G. K. Brian Underdahl Software Defined Networking For Dummies, 2015.

[10] T. S. Manar Jammala, Abdallah Shamia, RasoolAsalb, and Yiming Lic, "Software-Defined Networking: State of the Art and Research Challenges," Elsevier's Journal of Computer Networks.

[11] S. S. L. Jason Edelman, and Matt Oswalt, "Network Programmability and Automation", , Inc. , 1005 Gravenstein Highway North, Sebastopol, CA 95472.: O’Reilly Media, 2015.
[12] J. R. Nick Feamster, Ellen Zegura, ""The Road to SDN: An Intellectual History of Programmable Networks"."

[13] S. Azodolmolky, ""Software Defined Networking with OpenFlow"," 2013.

[14] Q. H. Fei Hu, and $\mathrm{Ke} \mathrm{Bao,} \mathrm{"Network} \mathrm{Innovation} \mathrm{through}$ OpenFlow and SDN Principles and Design"," 2014.

[15] O. N. Foundation, ""OpenFlow Switch Specificatio"," 2014.

[16] D. Samociuk, ""Secure Communication Between OpenFlow Switches and Controllers"," IARIA, 2015.

[17] M. t. P. tnoy, "Virtulization Essentials", Indiana: John Wiley \& Sons, Inc., Indianapolis, 2012.

[18] C. W. Gregor Schaffrath, “"Network Virtualization Architecture:Proposal and Initial Prototype"," ACM 978-1-60558595, 2009.

[19] M. K. C. a. R. Boutaba, ""A Survey of Network Virtualization"," 2008.

[20] R. J. a. S. Paul, "Network Virtualization and Software Defined Networking for Cloud Computing: A Survey"," IEEE Communications Magazine, 2013.

[21] Y. Y. Qiang Duan, and Athanasios V. Vasilakos, "A Survey on Service-Oriented Network Virtualization toward Convergence of Networking and Cloud Computing"," 2012.

[22] P. R. Konstantinos Samdanis, Andreas Maeder,Michela Meo and Christos Verikoukis, "GREEN COMMUNICATIONS PRINCIPLES, CONCEPTS AND PRACTICE": John Wiley \& Sons, Ltd, 2015.

[23] "Green Communications Theoretical Fundamentals, Algorithms and Applications": Taylor \& Francis Group, 2013.

[24] L. Haddon, ""Information and Communication Technologies in Everyday Life"," 2004.

[25] D. W. Mary Hayes, ""ICT in the Early Years"," 2006.

[26] S. B. Yadi Ma, ""Smart Pre-Classifier to Reduce Power Consumption of TCAMs for Multi-dimensional Packet Classification",' SIGCOMM'12, August 13-17, 2012, Helsinki, Finland.

[27] K. K. a. S. Banerjee2, ""Compact TCAM: Flow Entry Compaction in TCAM for Power Aware SDN"," 2014.

[28] S. S. Brandon Heller, Priya Mahadevan,Yiannis Yiakoumis,Puneet Sharma, ""ElasticTree: Saving Energy in Data Center Networks"," 2010.

[29] G. Y. Deze Zeng, Lin Gu, Song Guo, and Hong Yao, “"Joint Optimization on Switch Activation and Flow Routing towards Energy Efficient Software Defined Data Center Networks"," IEEE ICC, 2016. 